\title{
Control robusto de Oscilaciones en Sistemas de Potencia mediante Señales Remotas
}

\section{Robust Control of Oscillations in Power Systems by Remote Signals}

DOI: http://dx.doi.org/10.17981/ingecuc.12.2.2016.06

Artículo de Investigación Científica - Fecha de Recepción:23 de Mayo de 2016, Fecha de Aceptación: 21 de Agosto de 2016

\author{
Diego González Ocampo \\ Universidad Tecnológica de Pereira \\ Pereira, Colombia, diegogo@utp.edu.co
}

\author{
Alejandro Loaiza \\ Universidad Tecnológica de Pereira \\ Pereira, Colombia, alejoloaizar@hotmail.com
}

\author{
Alexander Arias Hernández \\ Universidad Tecnológica de Pereira \\ Pereira, Colombia, alarias@utp.edu.co
}

Cómo citar este artículo:

D. González Ocampo, A. Loaiza y A. Arias Hernández, "Control Robusto de Oscilaciones en Sistemas de Potencia Mediante Señales Remotas”, INGE CUC, vol. 12, No.2, pp. 58-67, 2016. DOI: http://dx.doi.org/10.17981/ingecuc.12.2.2016.06

\begin{abstract}
Resumen- Este artículo presenta una estrategia de control robusto que hace uso de Power System Stabilizers (PSS's) para enfrentar el problema de las oscilaciones interárea en sistemas eléctricos de potencia. La propuesta se fundamenta en la estrategia $\Delta \omega$ planteada por Kundur y adecuada para tener en cuenta mediciones remotas de todas las máquinas generadoras del sistema de potencia. El Centro de Control calcula una señal de control para cada generador del sistema ponderando las mediciones de cada generador activo. La robustez de la propuesta permite afrontar el problema de los retardos que se presentan en las señales de comunicaciones debido a las mediciones remotas. La fortaleza de la propuesta se evidencia mediante su implementación sobre el sistema de prueba de 2 áreas y 4 máquinas de Kundur, en la que se consideraron los modelos completos de las máquinas. Se logró amortiguar de manera satisfactoria las oscilaciones que surgen después de implementar una falla trifásica a tierra, también se manejó adecuadamente el retardo en las señales de comunicación las cuales agregan inestabilidad al lazo cerrado.
\end{abstract}

Palabras clave-Sistemas eléctricos de potencia; control de sistemas eléctricos; estabilidad de sistemas de potencia; latencias en comunicaciones; retardos en comunicaciones.

\begin{abstract}
This paper shows a robust control strategy to address the inter-area oscillations in power systems using PSS's. The approach is based on the well-known $\Delta \omega$ strategy of Kundur, and it was modified to take into account remote signals of the whole power system. The control center computes control signals for each generator in the system weighting the different measurements of each active generator. The robustness of the approach allows to face the communications delays due to remote measures. The strength of the proposed technique is validated through simulations over the Kundur two-area-fourmachines test case power system, here it was used the complete model for the generators. The inter-area oscillations after a three-phase fault were successfully damped; also, the latencies which add instability in closed loop were faced.
\end{abstract}

Keywords- Power systems; power systems control; power systems stability; latencies; communications delays; robust control. 


\section{INTRODUCCIÓN}

La seguridad y confiabilidad de los sistemas de potencia están estrechamente asociadas con su capacidad de mantenerse en una región estable con variables igualmente estables. Esta región estable es definida por valores acotados de los estados del sistema de potencia [1]. En la actualidad se tienen sistemas de potencia de enormes dimensiones en los que mantener la estabilidad se ha vuelto determinante y cada vez más complicado. En estos sistemas tan grandes, las oscilaciones que surgen después de ciertas contingencias pueden fácilmente llevar el sistema de potencia al colapso [1-3].

Para mantener la estabilidad de los sistemas de potencia se han diseñado múltiples estrategias orientadas, por un lado, a mejorar la estrategia de control y, por otro lado, hacer más sofisticado y confiable el monitoreo de los sistemas de potencia. En lo que tiene que ver con las estrategias de control, se han hecho aportes sobre el control de los generadores mediante el uso de estabilizadores de sistemas de potencia (Power System Stabilizers, PSS) alimentados con señales locales; así mismo, se ha mejorado cada vez más el diseño de los parámetros de los PSS [1],[3],[4]. En cuanto al monitoreo, se han implementado técnicas de análisis de información y hoy día se ha superado el uso de los esquemas SCADA, y se ha llegado a la implementación de las Phasor Measurement Units (PMU), que mejoran el desempeño de los Wide Area Measurement Systems (WAMS) [5-7].

En el caso específico de las oscilaciones interárea de sistemas de potencia se tienen aplicaciones basadas en señales medidas localmente $y$ controladores locales, pero la literatura ha demostrado que para el control de tales oscilaciones el uso de señales remotas es más efectivo [10], [11]. Ahora, aunque algunos autores han hecho uso de señales remotas para el control de oscilaciones interárea, se ha presentado el reto de mejorar el desempeño de tales estrategias de control de área amplia cuando las señales remotas llegan con considerables retardos al controlador general [12]-[14].

Este artículo presenta una estrategia de control robusta para enfrentar el problema de las oscilaciones interárea en sistemas de potencia y que considera la presencia de retardos en las señales de comunicaciones.

Este artículo está organizado de la siguiente forma: en la sección 2 se describen las generalidades del sistema de potencia, la forma en la que aparece el problema de las oscilaciones interárea, así como la consideración del problema del retardo en los sistemas de comunicaciones. En la sección 3 se presenta la propuesta denominada Delta-w-PM, y en la sección 4 se presenta los resultados de la aplicación de la propuesta de control de los autores. Finalmente se presenta algunas conclusiones relevantes so- bre el tema y la bibliografía. Cabe señalar que lo aquí presentado surge en el marco del proyecto de investigación "Estrategia de control no lineal de oscilaciones transientes en sistemas de potencia con señales retardadas" financiado por la Universidad Tecnológica de Pereira.

\section{Oscilaciones INTERÁREa y Presencia de Retardos En SeÑales}

\section{A. Descripción del problema de las oscilaciones interárea}

\section{1) Formación de sistemas de potencia densamente interconectados}

Las necesidades de energía eléctrica de un país usualmente son soportadas por distintas fuentes de generación que se conectan entre sí para garantizar confiabilidad y continuidad del servicio. Debido a la necesidad de mantener una confiabilidad elevada, los sistemas eléctricos usan diferentes enlaces eléctricos para llevar la energía al consumidor final, lo cual genera un sistema de potencia enmallado compuesto por interconexiones eléctricas densas [1, 2]. Lo anterior, aunque mejora la confiabilidad de los sistemas, incrementa la conectividad en todo el sistema y provoca que los eventos en una zona específica afecten la totalidad del sistema eléctrico.

En estos sistemas densamente interconectados se presentan cambios permanentes en las variables eléctricas. Tales variaciones son los cambios en los valores de demanda, las modificaciones en las cantidades de generación y en otros casos las congestiones o limitaciones térmicas para la transferencia de potencia $[1,2]$. Lo anterior indica que un sistema altamente interconectado es constantemente perturbado tanto por las variaciones pequeñas como por las debidas a contingencias, y estas variaciones se reflejan en todo el sistema de potencia.

\section{2) La formación de sistemas de área amplia}

Una vez se tienen sistemas eléctricos funcionales, estos pueden crecer en tamaño debido al creciente número de cargas eléctricas que aparecen en un sistema de potencia. Esto es, el crecimiento de cargas eléctricas puede producirse con la aparición de nuevos centros de consumo o con el incremento de la demanda de las cargas ya existentes.

Esta exigencia al sistema debido a las cargas se resuelve inicialmente mediante la instalación de nuevas centrales de energía, nuevas líneas o corredores eléctricos para fortalecer la interconexión. Esencialmente, el crecimiento de la demanda de energía implica el aumento de la complejidad operativa del sistema [1, 2].

Por otro lado, así como la formación de áreas cada vez más grandes puede generarse por el crecimien- 
to de los sistemas de potencia, estas grandes áreas también pueden aparecer debido a la integración de sistemas de potencia de diferentes áreas geográficas. En la Fig. 1 se muestra el sistema de prueba de 2 áreas de Kundur [1] que ejemplifica lo mencionado. En este sistema se ilustra la formación de 2 áreas eléctricas diferentes que básicamente representan dos áreas operativas, cada una con 2 generadores y con sus respectivas cargas. Las áreas mencionadas están unidas mediante líneas de conexión denominadas tie lines y que pueden verse conectando los nodos 7, 8 y 9. El sistema conformado por estas áreas que se interconectan para integrar sus capacidades de generación recibe el nombre general de sistemas de potencia de área amplia [1].

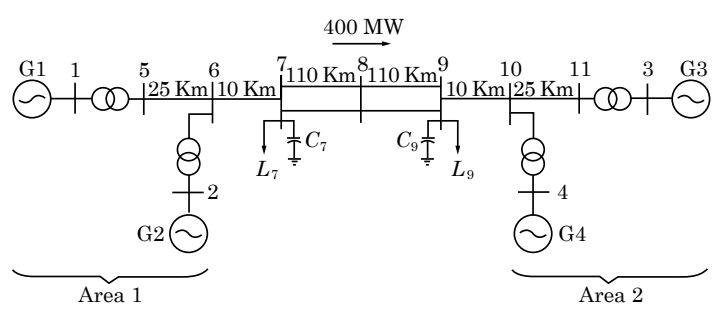

Fig. 1. Sistema de prueba de Kundur. Fuente: Tomado de [1].

\section{3) Los problemas fundamentales de los sistemas de área amplia}

Dentro de cada área operativa existen generadores con sus equipos de control, sus propias demandas de energía y sistemas de transmisión. Generalmente, los controladores usados se basan en el estabilizador del sistema de potencia (PSS), que actúa sobre los reguladores automáticos de voltaje (Automatic Voltage Regulators: AVR). Aquí los AVR juegan un destacado papel en el establecimiento de la tensión del sistema, mientras que para mantener la frecuencia dentro de los rangos deseados se utilizan los gobernadores de las turbinas [2].

Dado que en la misma área geográfica de los sistemas multimáquina existen varios generadores, las variaciones de tensión o de frecuencia mencionadas afectan en proporciones similares a cada generador dentro del área [2, 3]. De la misma forma, debido tanto a la cercanía eléctrica como a la fuerte conectividad de las máquinas de generación dentro de una misma área geográfica, los generadores actúan coherentemente manteniendo su frecuencia en valores muy cercanos [3, 4]. En algunos casos estas máquinas sufren una perturbación en su comportamiento que afecta la frecuencia; este efecto se distribuye en el resto de las máquinas de generación vecinas. Este fenómeno se conoce como oscilación intraárea. El sistema en conjunto descrito anteriormente puede formularse matemáticamente como

$$
\begin{aligned}
& \dot{x}(t)=f(t, x) \\
& y(t)=h(t, x)
\end{aligned}
$$

Esta formulación nos indica la existencia de un sistema no lineal que es variante en el tiempo y cuyo funcionamiento depende de sus estados internos [3].

Debido a la interconexión de áreas mediante las tie lines, las dos áreas se afectan entre sí debido a sus problemas internos. Esto es, en cada área puede presentarse una perturbación cuyos efectos pueden transferirse al resto de áreas. Este efecto se conoce como oscilación interárea. La oscilación interárea se refiere a la oscilación que relaciona a las diferentes áreas conectadas en un sistema multiárea. La oscilación se presenta en la potencia eléctrica transferida entre las áreas operativas [3, 8].

Debido a que la dinámica bajo estudio es una dinámica lenta (cuya frecuencia oscila entre 0.3-2 Hz), es posible usar esquemas de control lineales y efectuar el análisis del sistema usando su modelo linealizado alrededor de un punto de operación de interés $[1,2,8]$. Entonces, el modelo linealizado al rededor del punto de operación puede tener la siguiente forma:

$$
\begin{aligned}
& \dot{x}(t)=A x(t) \\
& \mathrm{y}(\mathrm{t})=\mathrm{Cx}(\mathrm{t})
\end{aligned}
$$

El anterior modelo matemático contiene los modos oscilantes y la descripción de los polos del sistema de potencia [3].

En los sistemas de potencia que presentan oscilaciones interárea existe un bajo valor de torque amortiguador en las máquinas generadoras. Por esto, las oscilaciones pueden incrementar su valor y derivar en excursiones demasiado grandes de los valores de separación angular. Esta separación angular se asocia directamente a oscilación de la potencia transferida en las tie lines entre áreas, como se mencionó anteriormente. Ahora, cuando existe la oscilación interárea en los valores de potencia, estas limitan la potencia transferida y pueden causar la actuación de las protecciones, lo que a la postre puede provocar el aislamiento de las diferentes áreas y un apagón generalizado en todo el sistema $[1,8]$.

\section{B. Descripción del impacto del retardo en los sistemas de potencia}

El retardo en las comunicaciones es definido como la diferencia de tiempo desde el momento en que la señal es medida hasta el momento en cual es utilizada por el controlador [9]. Lo anterior se presenta cuando las señales medidas viajan largas distancias en su proceso de transmisión, y es una característica que se presenta fundamentalmente cuando se requiere controlar sistemas de área amplia mediante el uso de señales remotas [10, 11].

La existencia del retardo (también llamada latencia), que es causado principalmente por la transmisión de datos a través de la red de comunicaciones, provoca que el controlador reciba una señal diferente de la señal real y que como resultado se tomen decisiones o acciones de control erradas [12]. Es decir, el 
controlador toma decisiones de control que no corresponden a la realidad operativa del sistema.

En la Fig. 2 se ilustra el concepto de una señal retardada. En este caso, una señal que luego de pasar por el medidor se entrega al destinatario con un retardo .

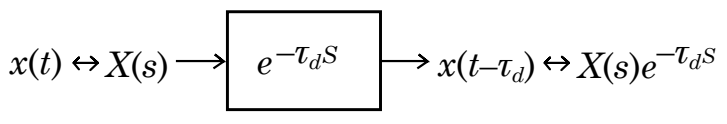

Fig. 2. Diagrama de bloques del desplazamiento de la señal debido al retardo. Fuente: Elaboración propia.

Puede notarse que a la salida del bloque de medición (en la figura 2) se obtienen señales desplazadas en el tiempo (también se representa el desplazamiento en la frecuencia). La Fig. 3 ilustra la presencia del retardo en el lazo cerrado de control. El retardo se incluye en el proceso de medición que se entrega al sistema de control, que a su vez es usado para controlar el sistema de potencia [13].

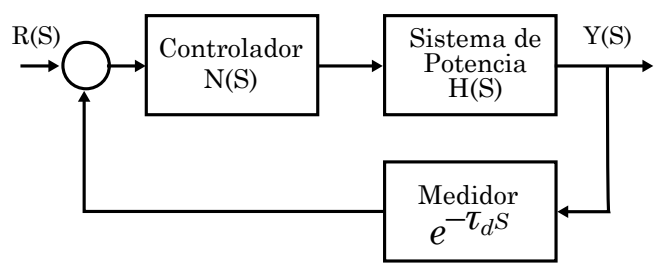

Fig. 3. Diagrama de bloques del control con retardos en mediciones. Fuente: Elaboración propia.

El diagrama de bloques de la Fig. 3 permite obtener la función de transferencia de lazo cerrado que se ilustra en la siguiente ecuación:

$$
H_{l c}(s)=\frac{N(s) H(s)}{N(s) H(s) e^{-\tau} d^{s}+1}
$$

Puede notarse que la función de transferencia se modifica con el valor del retardo en la frecuencia . En este caso, el valor del retardo altera el comportamiento del sistema en su conjunto [13,14].

Para este artículo, y con el propósito de ilustrar el impacto del retardo, se efectuaron simulaciones que incluían retardos en las señales de la medición. De esta forma se verificó la alteración de la función de transferencia y, por lo tanto, de la variación del comportamiento de los sistemas en lazo cerrado. Se tomó como referencia el sistema en lazo cerrado correspondiente al sistema Kundur de dos áreas. Este sistema fue sometido a una falla trifásica a tierra durante 5 ciclos en $\mathrm{t}=1 \mathrm{~s}$, la cual fue eliminada mediante la apertura de las líneas bajo falla. En tal sistema se procuró mantener la potencia entre las áreas en un valor fijo, se incluyeron los retardos y se obtuvieron los siguientes resultados [15]:

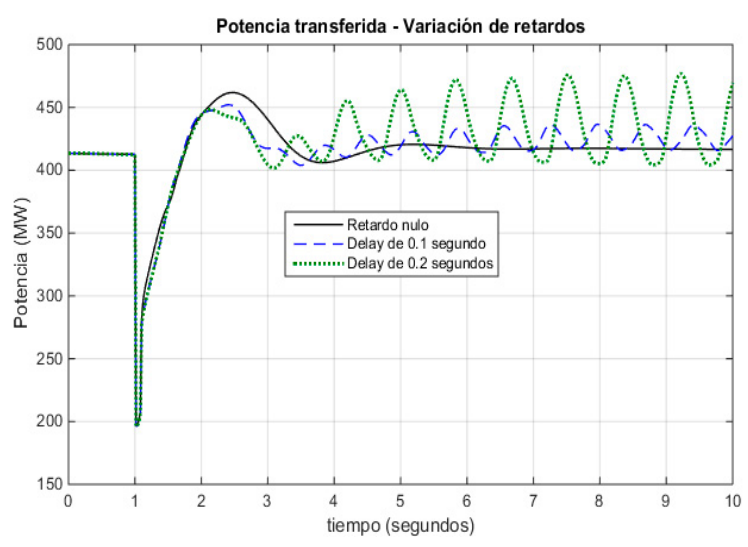

Fig. 4. Comportamiento de potencia transferida en presencia de retardos. Fuente: Elaboración propia.

En la Fig. 4 puede notarse lo siguiente: con un retardo nulo el sistema de control implementado actúa adecuadamente después de la falla. Con un retardo de 100 milisegundos empieza a notarse que el sistema pierde la habilidad de amortiguar las oscilaciones interárea y estas crecen paulatinamente. Finalmente, con un retardo de 200 milisegundos se hace evidente el gran impacto desestabilizador que tienen los retardos en el sistema de potencia. Así, es clara la necesidad de construir una metodología para el control de sistemas multiárea que pueda operar eficientemente a pesar de la presencia de retardos debido al uso de señales remotas.

\section{Formulación final del problema por resolver}

Tal como se ha descrito, el problema por resolver es el de las oscilaciones interárea en sistemas de potencia. Ya se ha mencionado que un modelo linealizado puede permitir tener la información suficiente. Ahora, para controlar tal problema se empleará las señales remotas, que permiten tener mejor visibilidad del problema en conjunto, de esta forma se tiene un sistema que en lazo cerrado puede representarse como

$$
\begin{gathered}
\dot{x}(t)=A x(t)+B u(y(t)) \\
y(t)=C x(t)
\end{gathered}
$$

En este momento la representación del sistema incluye la realimentación en lazo cerrado de las variables del sistema de potencia, y puede notarse que la señal de control es función de las salidas del sistema monitoreadas. Aquí es válido plantear una ley de control de realimentación lineal como $u(y(t))$ $=K_{d} y(t)$. El modelo anterior se obtiene alrededor de un punto de equilibrio. Teniendo en cuenta que estas mediciones remotas deben ser modeladas, y de acuerdo con lo ilustrado en la sección II.2 se tiene un nuevo modelo en el que la señal de control está siendo afectada por el retardo $\tau_{d}$ : 


$$
\begin{gathered}
\dot{x}(t)=A x(t)+B K_{d} y\left(t-\tau_{d}\right) \\
y(t)=C x(t)
\end{gathered}
$$

Ahora, teniendo en cuenta lo anterior, es necesario incluir los cambios del modelo debido a las variaciones paraméticas del punto de operación o a contingencias como fallas, apertura de líneas o pérdidas de demanda eléctrica. Eso hace que el modelo anterior se comporte como

$$
\begin{gathered}
\dot{x}(\mathrm{t})=(A+\Delta A) x(t)+(B+\Delta B) K_{d} y\left(t-\tau_{d}\right) \\
y(t)=(C+\Delta C) x(t)
\end{gathered}
$$

Donde los valores de describen las variaciones paraméticas y las incertidumbres del modelo. Es decir, el modelo anterior es un modelo linealizado que tiene en cuenta las incertidumbres en los parámetros debidas a un mal modelo. Así como tiene en cuenta las variaciones debidas a las fallas, también se incluye el efecto del retardo. Como puede verse, el sistema modelado implica la necesidad de un sistema de control robusto que pueda actuar no solamente sobre el problema nominal modelado en la ecuación (5), sino también el problema más realista modelado en la ecuación (6).

\section{III.Propuesta de Control Multiárea}

En la anterior sección se mostró que los sistemas de área amplia pueden controlarse mejor con señales remotas, pero que el uso de las señales remotas implica afrontar los retardos debido a la transmisión a grandes distancias. En el caso de no existir retardos, las oscilaciones interárea en un sistema de potencia pueden ser controladas satisfactoriamente. No obstante, al presentarse retardos la situación es diferente y el caso específico de un retardo de 100 milisegundos ha mostrado que el sistema pierde la capacidad de amortiguamiento de las oscilaciones interárea.

Considerando la situación anterior, en esta sección se propone una sencilla pero eficaz metodología para el control de las oscilaciones interárea en sistemas de potencia. La metodología propone un control en lazo cerrado mediante la realimentación de los valores de las salidas; es una especie de realimentación de lazo cerrado modificada y adaptada específicamente para el caso de los sistemas de potencia donde el reto fundamental es encontrar un valor para que permita controlar el sistema de potencia aun con las variaciones paramétricas y con los valores de retardo.

La metodología se diseña e implementa teniendo en cuenta las siguientes suposiciones:

- Existen equipos de medición en todas las máquinas de generación y en las variables de interés.

- El retardo de las mediciones tiene el mismo valor para todas las medidas e incluye el retardo de envío de las señales.
- Las medidas se concentran en un agente que toma las decisiones de forma centralizada y las envía a cada PSS que se encuentra en las unidades de generación.

\section{A. El sistema de potencia}

Se considera la existencia de sistemas de potencia con sus máquinas de generación, las líneas de interconexión y valores de demanda eléctrica que pueden variar en el tiempo. El caso considerado supone la existencia de una demanda constante durante el tiempo de simulación.

Las máquinas de generación tienen un gobernador y un AVR. A su vez, las máquinas están conectadas mediante líneas de alta tensión que se encargan de transmitir la potencia requerida dada la demanda. Para efectos de modelamiento se considera las cargas como demandas de potencia constante.

En el modelo se ha tenido en cuenta que en el caso de fallas los dispositivos de protección actúan para efectuar la apertura de las líneas y despejar tales fallas.

\section{B. Sistema de monitoreo y medición}

Una de las suposiciones para la implementación de la metodología es la existencia de elementos de medición en el sistema de potencia. Los sistemas modernos de medición superan la velocidad de los sistemas de medición tradicionales, dado que las unidades de medición fasorial (PMU's) y dispositivos similares son capaces de tomar lecturas a una tasa mayor que la de los sistemas SCADA. En la Fig. 5 se ilustra la diferencia entre el sistema SCADA y la medición PMU.

Para entender un poco más acerca del funcionamiento de una PMU puede consultarse la referencia [16].

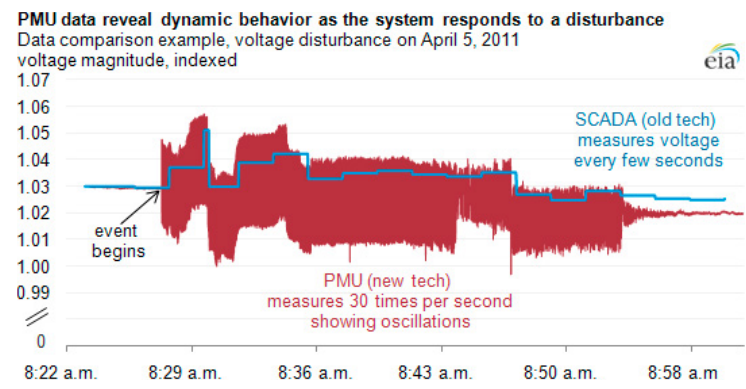

Fig. 5. Comparación de medidas de SCADA y PMU. Fuente: tomado de www.eia.gov

La existencia de elementos de medición de grandes sistemas de potencia implica un mejoramiento trascendental en el monitoreo de los mismos [6, 7,17]. Este tipo de medición o monitoreo de área amplia abre la posibilidad de implementar controles en lazo cerrado para mejorar el desempeño en la operación. 


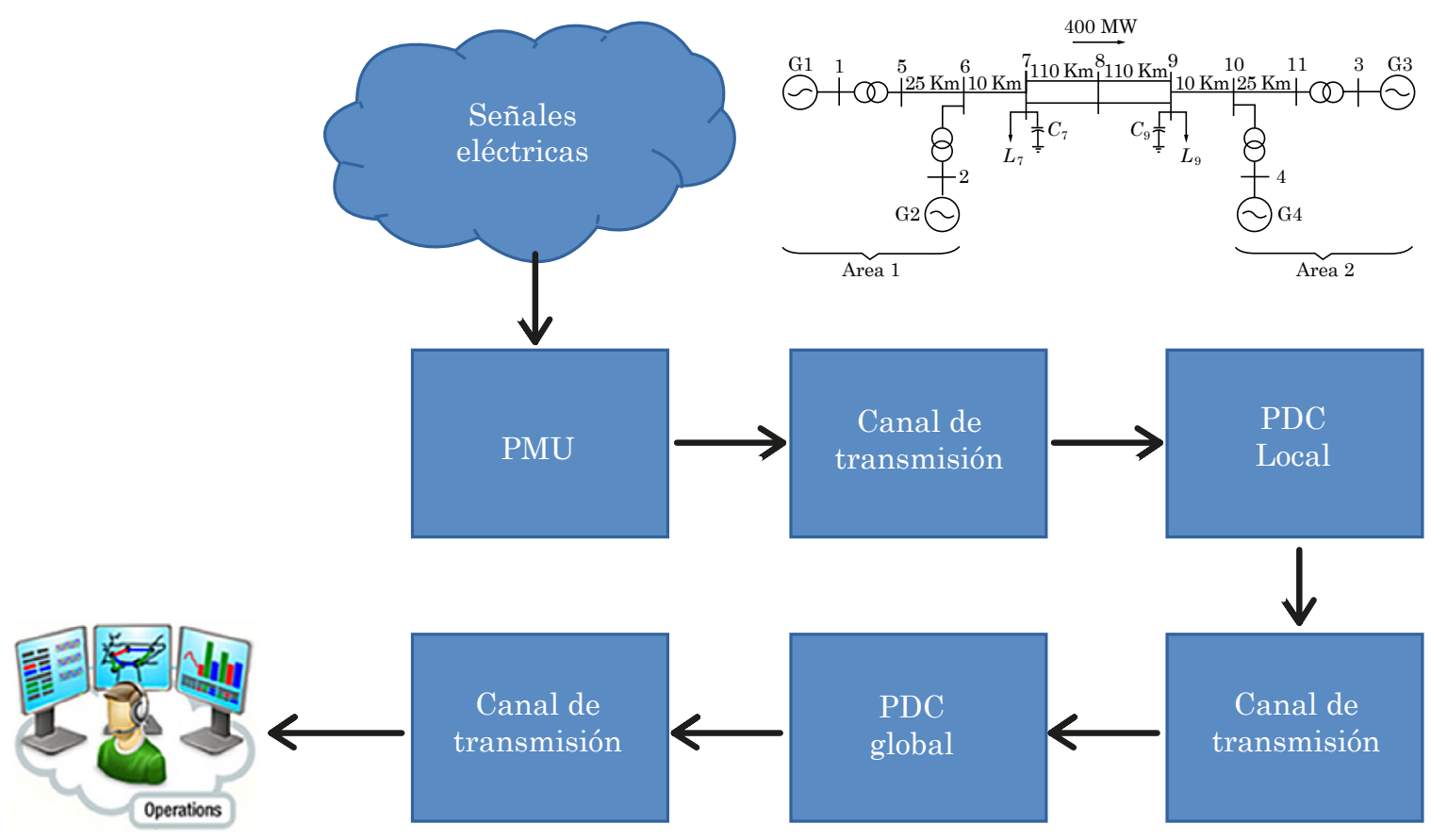

Fig. 6. Diagrama para ilustrar los componentes del retardo en las comunicaciones. Fuente: Tomado de www.eia.gov y [1] y modificado.

Sin embargo, la implementación de la medición de área amplia (Wide Area Measurement Systems: WAMS) tiene implícita la presencia de retardos en el tiempo de las señales transportadas $[6,18]$.

En los esquemas WAMS, cada medidor PMU toma lecturas y las envía a un concentrador denominado Phasor Data Concentrator (PDC), que a su vez las retransmite al controlador central en el sistema de potencia. Todo lo anterior implica una demora entre el momento de la lectura del medidor y el momento en el que llega la información al controlador central [18]. La Fig. 6 ilustra la situación mencionada.

En todo el proceso se presentan retardos que pueden representarse mediante la siguiente ecuación:

$$
\tau_{d}=\tau_{f j o}+\tau_{P M U}+\tau_{C T 1}+\tau_{P D C L}+\tau_{C T 2}+\tau_{P D C G}+\tau_{C T 3}(7)
$$

En tal medición $\tau_{f i j o}$ es el componente fijo del retardo del sistema; $\tau_{P M U}$ es el retardo asociado a la medición de la señal; $\tau_{C T 1}$ es el retardo asociado al canal de transmisión entre la PMU y el PDC local; $\tau_{P D C L}$ es el retardo agregado en el PDC local por procesamiento de la señal; $\tau_{C T 2}$ es el retardo asociado al canal de transmisión entre el PDC local y el PDC global, $\tau_{P D C G}$ es el retardo asociado al PDC global; $\tau_{C T 3}$ es el retardo asociado al canal de transmisión existente entre el centro de control y el PDC global. El valor total del retardo es entonces $\tau_{d}$, que se incluye en la ecuación 3 .

\section{Propuesta de control Delta-w-PM}

En el caso del problema del sistema de potencia se tiene un modelo lineal nominal como en la ecuación (5). Ahora, puede hacerse referencia a que ese modelo es un punto del espacio n-dimensional. Con lo anterior puede afirmarse que las perturbaciones e incertidumbres están contenidas en una zona dentro del mismo espacio n-dimensional y que contiene al modelo nominal, el modelo perturbado por las variaciones paramétricas y le modelo perturbado por el retardo: esta es llamada bola n-dimensional. Es así que en el campo de la teoría de control se define como un control robusto todo aquel control que es capaz de gestionar tanto el problema nominal como los problemas en la vecindad del punto nominal y contenidos dentro de la bola n-dimensional. Por tanto, la propuesta presentada es por sí misma una propuesta de control robusto para los sistemas de potencia, capaz de manejar las oscilaciones interárea con la apertura y cierre de líneas de potencia (cambios paramétricos) y aun con la presencia de retardos en el sistema de monitoreo.

La propuesta en la realimentación no es de los valores de las variables de estado, dado que es complejo obtenerlos en los sistemas de potencia. Esta propuesta se basa en la realimentación de las señales de potencia y de velocidad. Aquí emerge la primera adaptación de la propuesta. Por otro lado, contrario a otros esquemas de control, se ha considerado la necesidad de crear unas reglas para determinar el valor de la matriz de 
realimentación , y que actuará directamente sobre los PSS's.

Para implementar la propuesta se considera lo siguiente:

a. Existen equipos de medición $P M U$ en cada máquina de generación.

b. Existe un $A V R$ en cada máquina generadora.

c. Existe un controlador central que recibe la información de todas las mediciones tomadas en cada generador.

d. Existe un retardo entre 100 y 400 milisegundo pero que se mantiene constante durante la simulación. Este tiempo de retardo resume el tiempo total de retardo desde que se toma la señal en las máquinas hasta retornar con señales de control sobre el generador [16].

En la literatura [1-3] se ha descrito una metodología de control denominada $\Delta \omega$ (Delta w), basada en el monitoreo de las variaciones de velocidad de las máquinas. Estas variaciones son enviadas al PSS, que posteriormente entrega una señal de control al AVR [1]. Este trabajo toma como base la propuesta $\Delta \omega$ (Delta w) de Kundur y se le efectúa modificaciones para poder actuar con señales remotas. Es decir, la propuesta Delta w es modificada y mejorada para que pueda actuar basada en las mediciones de área amplia [15] [18].

La propuesta es básicamente la siguiente: todas las medidas son tomadas por un concentrador central, como se ilustra en la Fig. 7. El controlador central toma los valores medidos mediante el sistema WAMS y a partir de ello calcula una señal que alimentará los PSS en cada máquina de generación. Para obtener cada medida registrada por el sistema WAMS es ponderada o castigada de forma que la señal obtenida es una especie de suma ponderada que contiene información de todo el sistema de potencia. Esta señal es denominada señal ponderada del PSS y matemáticamente se calcula con

$$
P S S_{j}=\sum_{\forall i}\left(k_{i j} \cdot l_{i j} \cdot \Delta w_{i}\right)
$$

Aquí representa la desviación de la velocidad, es el valor de ponderación, es un factor de acople entre la señal medida en la máquina $i$ y el PSS en la máquina $j$. En la expresión anterior, el índice toma valores de -1 o 1 . La ponderación que se realiza debe ser tal que la suma completa de valores de sea unitaria, lo cual se representa como

$$
\sum_{\forall i} k_{i j}=1
$$

Para seleccionar los diferentes valores de $k_{i} \mathrm{y}$ de $l_{i j}$ se deben seguir los siguientes pasos: i) los valores deben ser positivos menores que 1 , ii) los valores de $k_{i j}$ deben ser mayores que 0.6 y menores que 0.8 si $i=$ $j$; de esta forma, se garantiza que la mayor influencia sobre la máquina $j$ sea ella misma, iii) los valores de $l_{i j}$ deben ser 1 si $i=j$, y deben ser -1 en caso contrario. De esta forma todas las entradas de las otras máquinas tendrán influencia negativa [12].
Para seleccionar los diferentes valores de $k_{i}$ y de $l_{i j}$ se pueden seguir las siguientes recomendaciones:

$$
\begin{aligned}
& k_{i j}= \begin{cases}0 \leq k_{i j} \leq 1 & \text { si } i \neq j \\
0.6 \leq k_{i j} \leq 1 & \text { si } i=j\end{cases} \\
& l_{i j}=\left\{\begin{array}{cc}
-1 & \text { si } i \neq j
\end{array}\right. \\
& 1 \text { si } i=j
\end{aligned}
$$

En este sentido, se garantiza que la mayor influencia sobre la máquina $j$ sea ella misma. Además, todas las entradas de las otras máquinas tendrán influencia negativa.

En resumen, las señales se toman en cada generador, posteriormente se llevan a un controlador central que se encarga de recolectar toda la información para entregarla posteriormente a cada una de las máquinas controladas, es básicamente una realimentación de salidas en lazo cerrado ponderada por la Matriz $K_{d}$. Por esta razón, la propuesta mencionada recibe el nombre de Delta w Ponderado Multiárea o Delta-w-PM o $\Delta \omega$-PM [15].

En la Fig. 7 se ilustra la forma en la que actúa el esquema de control propuesto.

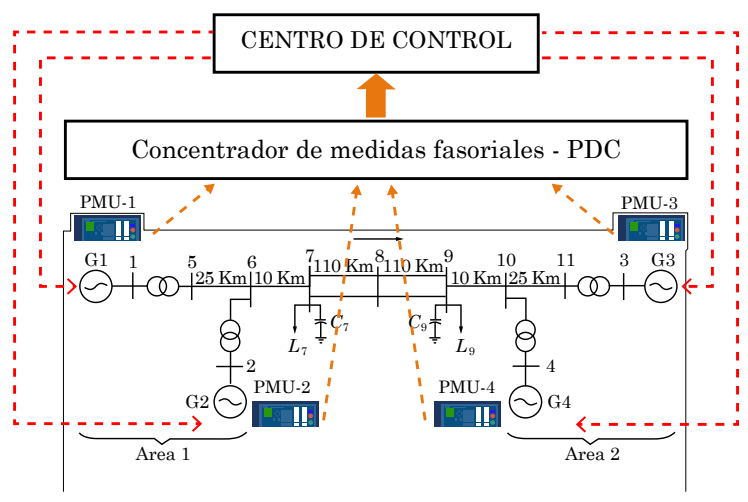

Fig. 7. Propuesta de control Delta-w-PM. Fuente: Tomado de www.sielec.com y [1] y modificado.

La propuesta fue implementada mediante el uso de Simulink ${ }^{\circledR}$ y SimpowerSystems ${ }^{\circledR}$. Esta propuesta puede generalizarse e implementarse sobre sistemas de potencia de mayor tamaño debido a su sencillez conceptual y su fácil implementación [15].

\section{Resultados Comparativos DeL Control Multiárea}

Normalmente en la literatura es común encontrar el uso del PSS para controlar las máquinas eléctricas del sistema de potencia. La Fig. 8 muestra el controlador utilizado:

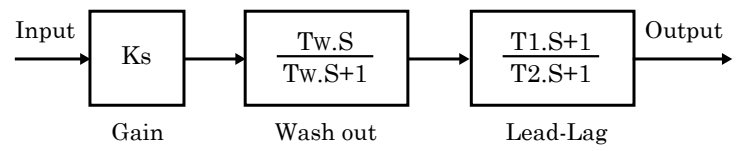

Fig. 8. PSS Delta w básico. Fuente: Tomado de [1] 
Para el controlador mostrado en la figura 8, la función de transferencia es

$$
\begin{aligned}
& H_{P S S}(s)=K_{S} \underline{T}_{w}\left(T_{1} \underline{\mathrm{S}}+1\right) \\
& \left(T_{w} S+1\right)\left(T_{2} S+1\right)
\end{aligned}
$$

Donde los términos $K_{S}, T_{w}, T_{1}, T_{2}$ se seleccionan de acuerdo con los parámetros de las máquinas y del sistema eléctrico según lo presenta Kundur en [1]. Estos dispositivos que se instalan en cada una de las máquinas transmiten una senal de control a los AVR y regulan la tensión en los terminales.

\section{A. Sistema de prueba}

El sistema utilizado para realizar las respectivas pruebas es el sistema de dos áreas de Kundur con líneas de conexión entre áreas de longitud de $220 \mathrm{~km}$ [1]. Todos los generadores tienen una potencia nominal de 900 MVA y una tensión nominal de $20 \mathrm{kV}$, cargas L7 y L9, con potencias consumidas de 950 y $1780 \mathrm{MW}$, respectivamente. Este sistema se muestra en la figura 1 . Se analiza la potencia transferida desde el área 1 hacia el área 2 por las líneas que conectan los nodos 7-9. El sistema se implementa en Simulink ${ }^{\circledR}$, con lo cual se tuvo la posibilidad de incluir el modelo completo de las máquinas de generación, demandas y líneas de transmisión. Esto hace más confiable la simulación efectuada.

En condiciones de operación normales, se simula una falla en una de las líneas que conectan las dos áreas (falla entre los nodos 7-9 trifásica a tierra). Dicha falla se presenta en $t=1 \mathrm{~s}$ y tiene una duración de 83 milisegundos ( 5 ciclos de una señal de $60 \mathrm{~Hz}$ ); allí las protecciones actúan dejando el sistema en funcionamiento con una sola de las líneas.

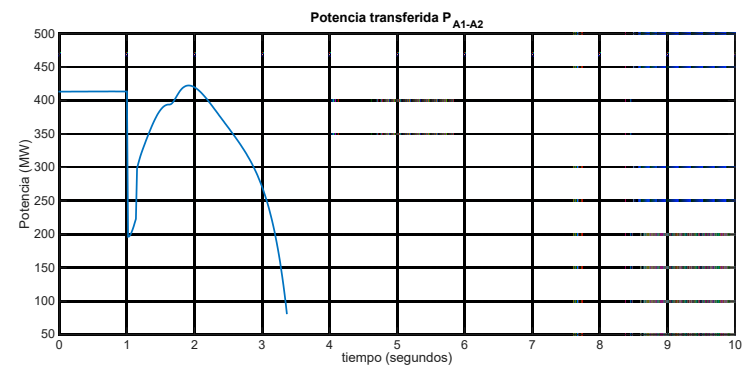

Fig. 10. Potencia transferida en presencia de perturbaciones sin sistema de control. Fuente: Elaboración propia.

La Fig. 10 muestra el comportamiento del sistema sin ningún tipo de control. Allí se nota que luego de ocurrida la perturbación, el sistema trata de recuperar su estado de operación normal, mas no es posible, y se presenta inestabilidad total.

\section{B. Sistema controlado con PSS Delta $w$ sin retardos}

En este caso se presentan los resultados del uso del control utilizado en la literatura sin la consideración de retardos en la señal [1]. Se puede apreciar que el sistema, luego de ocurrida la falla, recupera su estado de operación después de algunas oscilaciones, estabilizándose alrededor de los 5 segundos, como se nota en la Fig. 11. Sin embargo, el primer pico de potencia es crítico al ser tan alto, alrededor de 470 MW sobre los 2.5 segundos, y puede hacer que las protecciones se activen aislando los dos sistemas.

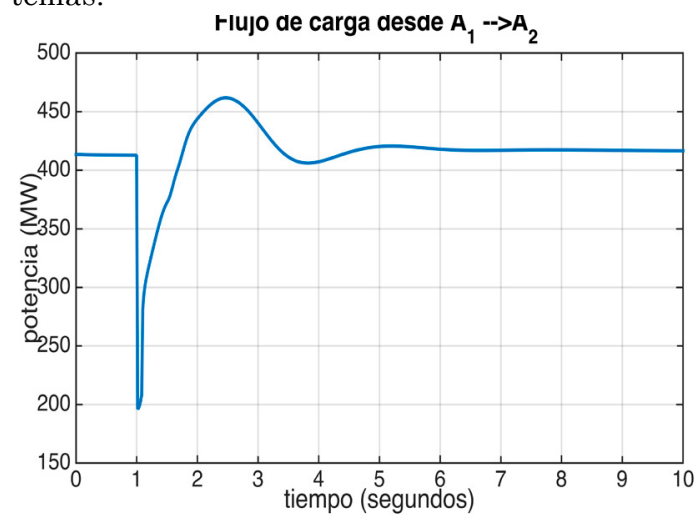

Fig. 11. Potencia transferida en presencia de falla trifásica con Control Delta w clásico [1]. WAMS sin retardos. Fuente: Elaboración propia.

\section{Implementación de la propuesta Delta-w- multiárea sin retardos}

Al implementar la propuesta Delta-w-PM se aprecia que los picos de las oscilaciones son menos pronunciados respecto del control clásico Delta-w. Lo anterior nos muestra que el desempeño de la propuesta es bastante prometedor.

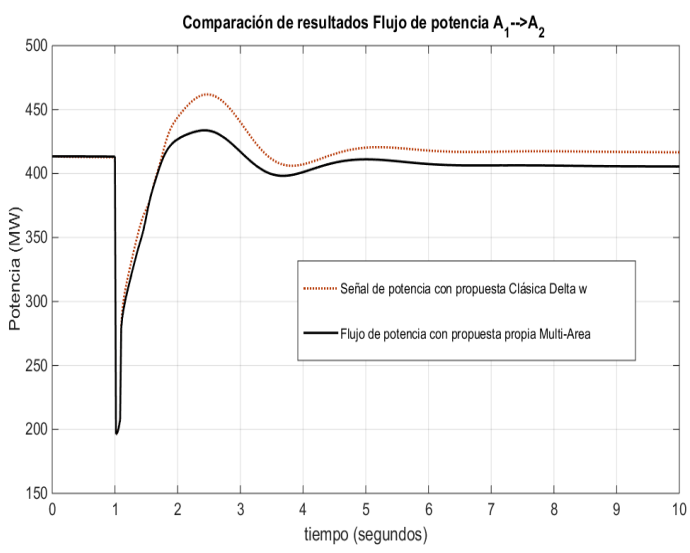

Fig. 12. Potencia transferida en presencia de perturbaciones. Comparación entre propuesta Delta w y Delta-w-PM. Fuente: Elaboración propia. 
Se puede ver que con el controlador Delta-w hay un incremento muy fuerte en la potencia, el pico es alrededor de $470 \mathrm{MW}$, que puede afectar los sistemas de protección obligándoles a actuar. En cambio, el controlador propuesto por los autores Delta-w-PM presenta mejores resultados tanto en la potencia pico (427 MW) como al momento de estabilizar el sistema, lo cual es apropiado en los sistemas de potencia. La propuesta elaborada por los autores y llamada Delta-w-PM produce respuesta con un menor valor de sobreimpulso y estabiliza el sistema un poco más rápido.

\section{Sistema controlado con PSS Delta-w con retardos de 100 milisegundos}

Aquí se realizan las pruebas teniendo en cuenta los retardos que se presentan por los sistemas de comunicaciones. Este retardo es el tiempo que toma la señal en ir desde su punto de medición hasta el centro de control. Para un sistema de área amplia, un valor de retardo típico es de 100 milisegundos.

La Fig. 13 muestra que el control Delta-w no es suficiente para hacer frente a los problemas de estabilidad que pueden ocurrir considerando los retardos que se presentan en las señales. Esto es un caso hipotético, dado que las señales de la propuesta Delta-w se toman localmente.

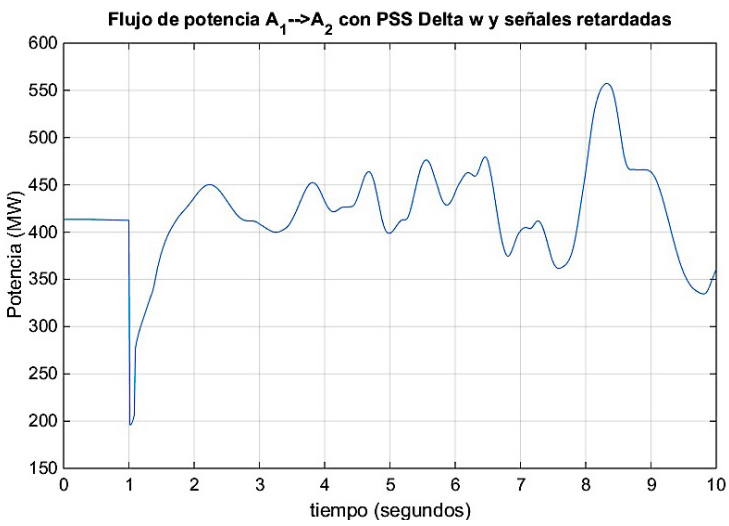

Fig. 13. Control Delta-w con señales retardadas. Fuente: Elaboración propia.

En la misma Fig. 13 puede verse que el sistema comienza inestable a partir de su primera gran excursión; evidentemente, tal situación es inaceptable en términos de confiabilidad y seguridad, puesto que este tipo comportamiento activa las protecciones y aislaría las dos áreas.

\section{E. Robustez al retardo del control propuesto Delta- $w-P M$}

Al implementar la propuesta desarrollada por los autores (Delta-w-PM) para tratar de afrontar un retardo de 100 milisegundos, se puede ver la robustez. El sistema es capaz de estabilizarse a los 3 segundos luego de ocurrida la falla. Este comportamiento se detalla en la Fig. 14. De esta manera, se soluciona el problema que generan los retardos en la señal y también se verifica que los picos de potencia no son tan elevados.

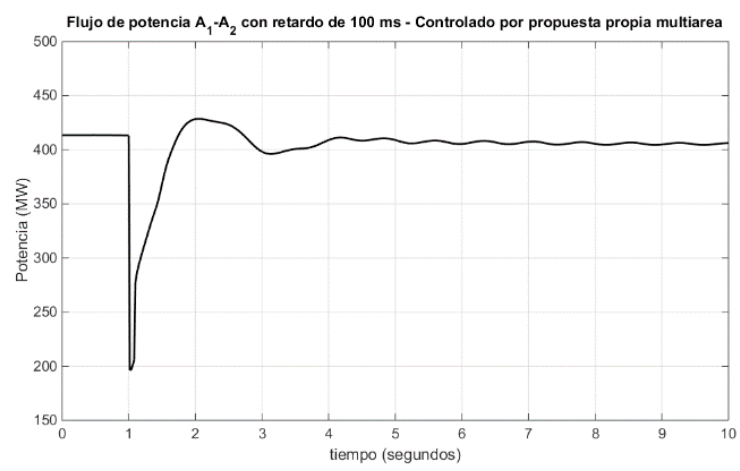

Fig. 14. Respuesta del sistema controlado por Delta-w-PM con retardo de 100 milisegundos. Fuente: Elaboración propia.

Finalmente, en la Fig. 15 se muestra el comportamiento de la transferencia de potencia cuando el tiempo de retardo se incrementa en un valor de 400 milisegundos. Con esta prueba se verificó el límite superior al cual el sistema respondía adecuadamente. Lo anterior quiere decir que si bien la propuesta es robusta para valores cercanos a los 100 milisegundos, puede fallar con valores mayores a $400 \mathrm{mi}-$ lisegundos.

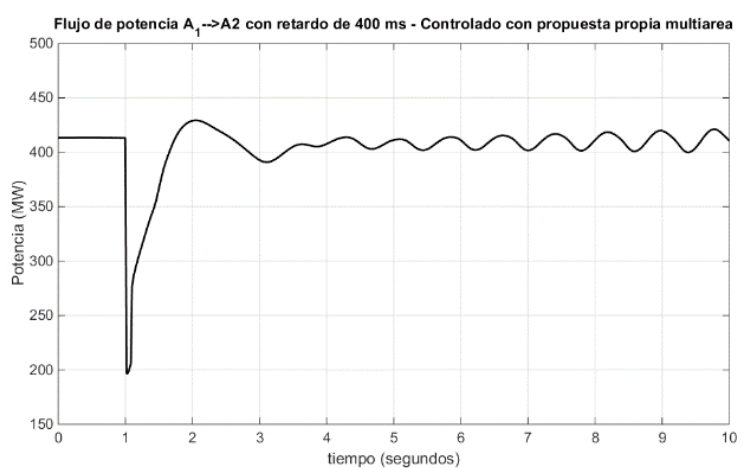

Fig. 15. Respuesta del sistema controlado por Delta-w-PM con retardos de 400 milisegundos. Fuente: Elaboración propia.

El valor de 400 milisegundos se convierte así en el límite superior para la robustez de la propuesta Delta-w-PM en el caso de este sistema de prueba específico. Aun así, los resultados son promisorios y pueden extenderse a sistemas de potencia más grandes. 


\section{Conclusión y Recomendaciones}

Se diseñó una propuesta de control robusta basada en la realimentación de señales de salida, la cual es capaz de enfrentar los retardos en sistemas de potencia de área amplia y que utilizan senales remotas.

Se verificó que los retardos en los sistemas de potencia pueden causar inestabilidades o comportamientos oscilatorios. Este retardo se implementó basado en los tiempos típicos de retardo descritos en la literatura; asimismo, se describió las componentes tecnológicas del sistema de comunicaciones responsables de los retardos de las comunicaciones en sistemas de potencia.

Se encontró que la propuesta propia Delta-wPM, basada en la presencia de WAMS, controla adecuadamente el sistema de prueba presentado. Se verifica así que el controlador es robusto frente a la existencia de retardos en las señales obtenidas de forma remota, y también es robusto frente a variaciones paramétricas debido a fallos en líneas y apertura de las mismas. La estrategia clásica Delta-w $(\Delta \omega)$, basada en la velocidad, falla cuando el retardo es de 100 milisegundos o menor. La estrategia Delta-w-PM presentada en este artículo es robusta para retardos inclusive de 400 milisegundos. Lo anterior es prometedor dado que es un problema actual documentado ampliamente como reto que debe ser superado.

Puede verse así mismo que un sistema de control que se alimenta con señales remotas obtenidas con sistemas WAMS funciona mejor que el control alimentado únicamente con señales locales. Esto es, las propuestas clásicas implementan un control usando señales locales de la velocidad y otras variables, y actúan solamente en las variables locales. En cambio, la propuesta Delta-wPM utiliza las señales de todas las máquinas para efectuar el control en todos los generadores.

\section{FinANCIACIÓN}

Artículo de investigación científica derivado del proyecto de investigación titulado "Estrategia de Control No-Lineal de Oscilaciones Transientes en Sistemas Eléctricos de Potencia con Señales Retardadas" de la Universidad Tecnológica de Pereira.

\section{REFERENCIAS}

[1] P. Kundur, Power system stability and control. New York: McGraw-Hill, 1994.

[2] M. Jan and B. Janusz, Power System Dynamics: Stability and Control. London: Wiley, 2009

[3] P. Kundur, M. Klein, and G. Rogers, "A fundamental Study of inter-area oscillations in power systems", IEEE Transaction on Power Systems, vol. 6, n 3, pp. 914-921, 1991.

[4] J. H. Chow and G. N. Taranto, "Robust Redesign of Power System Damping Controllers", IEEE Transactions on Control Systems Technology, vol. 3, n 3, pp. 290-297, September 1995.

[5] D. Bailey and E. Wright, Practical Scada for Industri, vol. 1. Burlington: Newnes, 2003.

[6] W. Mittelstad, P. Krause, P. Overholt, and J. Hauer, "The DOE Wide Area Measurement System (WAMS) project Demonstration of dynamic information technology for the future power system", in Proceedings on Fault and Disturbances Analysis/Precise measurements in Power Systems Conference, Arlington, 1995.

[7] M. Chenine and L. Nordstrom, "Modeling and Simulation of Wide-Area Communication for Centralized PMU-Based Applications", IEEE Transactions on Power Delivery, vol. 26, n³, pp. 1372-1380, 2011.

[8] P. Kundur, J. Paserba, V. Ajjarapu, G. Anderson, A. Bose, and C. Canizares, "Definition and Classification of Power System Stability", IEEE Transaction on Power Systems, vol. $19, \mathrm{n}^{\circ} 2$, pp. 1387-1401, 2004

[9] F. Milano and M. Anghel, "Impact of Time Delays on Power System Stability", IEEE Transactions on Circuits and Systems, vol. 59, $\mathrm{n}^{\circ} 4$, pp. 889-900, 2012.

[10] A. Aboul, A. Fouad, and A. Allam, "Damping controller design for power system oscillations using global signals", IEEE Transaction on power systems, vol. $11, \mathrm{n}^{\circ} 2$, pp. 767 $773,1996$.

[11] A. Snyder, D. Ivanescu, N. HadjSaid, D. Georges, and T. Margotin, "Delayed-input wide-area stability control with synchronized phasor measurements and linear matrix inequalities", in IEEE Power Engineering Society Summer Meeting, Seattle, 2000.

[12] H. Wu, K. Tsakalis, and G. Heydt, "Evaluation of Time Delay Effects to Wide-Area Power System Stabilizer Design", IEEE Transactions on Power Systems, vol. 19, $\mathrm{n}^{\circ} 4$, pp. 1935-1941, 2004.

[13] B. Naduvathuparambil, M. Valenti, and A. Feliachi, "Communication delays in wide area measurement systems", in Proceedings of the Thirty-Fourth Southeastern Symposium on System Theory, 2002.

[14] S.-I. Niculescu, Systèmes à retard. Aspects qualitatifs sur la stabilité et la stabilisation, París: Diderot Multimedia, 1997.

[15] A. Loaiza, "Implementación de control en sistemas de potencia de área amplia con medidas retardadas", Pereira, 2016.

[16] Y. Hu, V. Madani, R. Moraes, and D. Novosel, "Requirements of large-scale wide area monitoring, protection and control systems", in Proceedings on Fault Disturbance Analysis Conference, Atlanta, 2007.

[17] A. Bosé, "Smart Transmission Grid Applications and Their Supporting Infrastructure", IEEE Transaction on Smart Grid, vol. 1, n 1, pp. 11-19, 2010.

[18] D. Cai, Wide Area Monitoring, Protection and Control in the Future Great Britain Power System. Londres: Universidad de Manchester, 2012. 